\title{
On Numerical Computation of Pickands Constants
}

\author{
Hyemi Choi ${ }^{1, a}$ \\ ${ }^{a}$ Department of Statistics (Institute of Applied Statistics), Chonbuk National University, Korea
}

\begin{abstract}
Pickands constant $\mathcal{H}_{\alpha}$ appears in the classical result about tail probabilities of the extremes of Gaussian processes and there exist several different representations of Pickands constant. However, the exact value of $\mathcal{H}_{\alpha}$ is unknown except for two special Gaussian processes. Significant effort has been made to find numerical approximations of $\mathcal{H}_{\alpha}$. In this paper, we attempt to compute numerically $\mathcal{H}_{\alpha}$ based on its representation derived by Hüsler (1999) and Albin and Choi (2010). Our estimates are compared with the often quoted conjecture $\mathcal{H}_{\alpha}=1 / \Gamma(1 / \alpha)$ for $0<\alpha \leq 2$. This conjecture does not seem compatible with our simulation result for $1<\alpha<2$, which is also recently observed by Dieker and Yakir (2014) who devised a reliable algorithm to estimate these constants along with a detailed error analysis.
\end{abstract}

Keywords: extremes, Gaussian process, Pickands constant, fractional Brownian motion, DaviesHarte algorithm

\section{Introduction}

In probability and statistical theory Gaussian processes play an essential role by which many natural phenomena are reasonably modelled since a Gaussian process model can be specified by expectations and covariances; subsequently, its estimation and inference are relatively simple. Their applications are extremely numerous and diverse. Hence, the extremes of Gaussian processes have been investigated by many authors.

Let $\{\xi(t)\}_{t \in[0, h]}$ be a continuous centered stationary Gaussian process with covariance function $r(t)=\operatorname{Cov}\{\xi(s), \xi(s+t)\}$ that satisfies

$$
r(t)<1 \quad \text { for } t \in(0, h] \text { and } \quad r(t)=1-C|t|^{\alpha}+o\left(|t|^{\alpha}\right) \quad \text { as } t \rightarrow 0,
$$

where $h>0, \alpha \in(0,2]$ and $C>0$ are constants. It is well-known (Leadbetter et al., 1983; Pickands, 1969; Qualls and Watanabe, 1972) that there exists a constant $\mathcal{H}_{\alpha} \in(0, \infty)$ such that

$$
\lim _{u \rightarrow \infty} \frac{e^{\frac{u^{2}}{2}}}{u^{\frac{2}{\alpha}-1}} \mathrm{P}\left\{\sup _{t \in[0, h]} \xi(t)>u\right\}=\frac{h C^{\frac{1}{\alpha}}}{\sqrt{2 \pi}} \mathcal{H}_{\alpha} .
$$

$\mathcal{H}_{\alpha}$ is Pickands constant, the value of which depends on $\alpha$ only. Recently, Albin and Choi (2010) give a new and direct proof of the result (1.2) originally obtained by Pickands (1969), where as a by-product the following representation for Pickands constant $\mathcal{H}_{\alpha}$ by Hüsler (1999) is rediscovered.

\footnotetext{
This research was supported by Basic Science Research Program through the National Research Foundation of Korea (NRF) funded by the Ministry of Education, Science and Technology (NRF-2012R1A1A3010532).

${ }^{1}$ Department of Statistics (Institute of Applied Statistics), Chonbuk National University, Jeonju, Jeonbuk 561-756, Korea. E-mail: hchoi@jbnu.ac.kr
} 
Theorem 1. If (1.1) holds, then (1.2) holds with the constant $\mathcal{H}_{\alpha}$ given by

$$
\mathcal{H}_{\alpha}=\lim _{a \downarrow 0} \frac{1}{a} \mathrm{P}\left\{\max _{k \geq 1}\left(B_{\alpha}(a k)-(a k)^{\alpha}\right)+\eta \leq 0\right\}
$$

where $B_{\alpha}(t)$ is a fractional Brownian motion $(f B m)$ with a self-similar index $\alpha / 2$ and $\mathrm{E}\left\{B_{\alpha}^{2}(1)\right\}=2$, and $\eta$ is a unit mean exponentially distributed random variable that is independent of the process $B_{\alpha}$.

The constant $\mathcal{H}_{\alpha}$ appears in the extremal results of Gaussian processes and is also involved in the ruin probability $\left.\mathrm{P}\left\{\sup _{t \geq 0}\left(B_{\alpha}(t)-t\right)>u\right)\right\}$ often applied in telecommunication and insurance mathematics. However, the exact values of Pickands constants $\mathcal{H}_{\alpha}$ are known for $\alpha=1,2$, i.e., $\mathcal{H}_{1}=1, \mathcal{H}_{2}=1 / \sqrt{\pi}$ but unknown for other values of $\alpha$. Adler (1990) even said that it is unlikely to calculate Pickands constants. Much effort to find numerical approximations of $\mathcal{H}_{\alpha}$ has been made (see for example Shao (1996) and Burnecki and Michna (2002)). Dieker and Yakir (2014) recently estimated $\mathcal{H}_{\alpha}$ by simulation based on their new representation

$$
\mathcal{H}_{\alpha}=\mathrm{E}\left[\frac{\sup _{t \in \mathbb{R}} e^{B_{\alpha}(t)-|t|^{\alpha}}}{\int_{-\infty}^{\infty} e^{B_{\alpha}(t)-|t|^{\alpha}} d t}\right]
$$

along with a detailed error analysis and Harper (2014) proved that an often quoted conjecture $\mathcal{H}_{\alpha}=$ $1 / \Gamma(1 / \alpha)$ is false for small $\alpha$.

In this paper we utilize our expression of $\mathcal{H}_{\alpha}$ given in Theorem 1 to compute Pickands constant numerically. The numerical computation of $\mathcal{H}_{\alpha}$ requires the simulation of fBm. Hence we first review basic properties of $\mathrm{fBm}$ and introduce the Davies-Harte algorithm to simulate $\mathrm{fBm}$ in Section 2. The simulation results of Pickands constant are presented in Section 3. As concluding remarks we discuss and compare our results with Dieker and Yakir (2014) in Section 4.

\section{Preliminaries}

\subsection{Basic properties of fractional Brownian motion}

The family of fractional Brownian motions is indexed by a parameter $\alpha \in(0,2)$ where $H=\alpha / 2$ is called self-similarity or Hurst parameter. The self-similarity of the fBm $B_{\alpha}$ means $B_{\alpha}(a l) \stackrel{d}{=} a^{\alpha / 2} B_{\alpha}(l)$ for any $a>0$ in the sense of finite dimensional distributions. The process $B_{\alpha}$ has mean $\mathrm{E}\left\{B_{\alpha}(t)\right\}=0$ and covariances $\operatorname{Cov}\left\{B_{\alpha}(t), B_{\alpha}(s)\right\}=\left(\sigma^{2} / 2\right)\left(|s|^{\alpha}+|t|^{\alpha}-|s-t|^{\alpha}\right)$. Note that the increments of $B_{\alpha}$,

$$
Y_{j}=B_{\alpha}(j)-B_{\alpha}(j-1), \quad j=1,2, \ldots,
$$

form a stationary sequence called a fractional Gaussian noise (fGn), i.e., a normal random sequence with mean 0 and covariances

$$
\gamma_{\alpha}(j)=\frac{\sigma^{2}}{2}\left(|j+1|^{\alpha}+|j-1|^{\alpha}-2|j|^{\alpha}\right) \quad \text { for } j \in \mathbb{Z} .
$$

Then it follows from the self-similarity of $B_{\alpha}$ and the definition of $Y_{j}$ that

$$
B_{\alpha}(a l) \stackrel{d}{=} a^{\frac{\alpha}{2}} B_{\alpha}(l) \stackrel{d}{=} a^{\frac{\alpha}{2}} \sum_{j=1}^{l} Y_{j}
$$



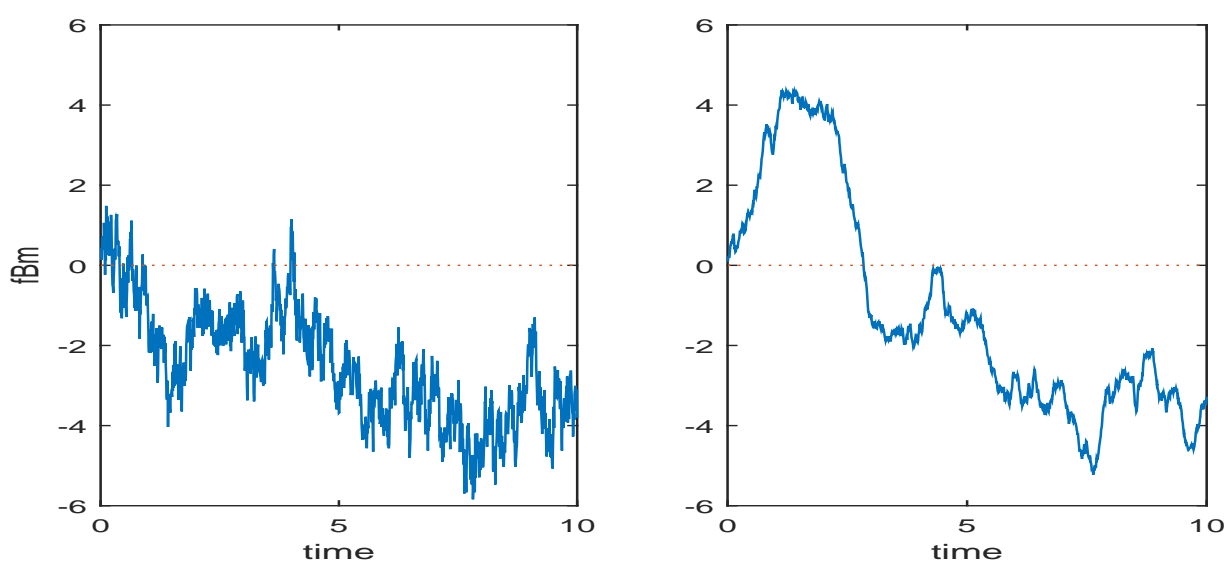

Figure 1: Sample paths of $B_{\alpha}$ (left: $\alpha=0.6$, right: $\left.\alpha=1.4\right)$.

The $B_{\alpha}$ is the only Gaussian self-similar process with stationary increments and hence a very important generalization of Brownian motion. For more details see Samoradnitsky and Taqqu (1994). The parameter $\alpha$ determines the extent of the dependence in the fGn with $\alpha=1$ corresponding to independence and $1<\alpha \leq 2$ to long range dependence. Note that $\left.\gamma_{\alpha}(j) \sim \sigma^{2} \alpha(\alpha-1)\right) j^{\alpha-2} / 2$ as $j \rightarrow \infty$ for $\alpha \neq 1$. Thus in case that $0<\alpha<1, \gamma_{\alpha}(j)$ are negative for large $j$. A realization of $B_{1.4}$ with $\sigma^{2}=2$ is shown in Figure 1 together with a realization of $B_{.6}$. The sample path of $B_{1.4}$ is relatively smooth since $\gamma_{\alpha}$ show a long range dependence for $1<\alpha \leq 2$, while the $B_{.6}$ case is rather erratic by the negativity of $\gamma_{\text {.6. }}$. Note that we assume $\sigma^{2}=2$ without loss of generality in the following.

\subsection{Simulation of fractional Brownian motion}

Self-similarity property can be used to obtain an $\mathrm{fBm}$ sample on an arbitrary equispaced grid by computing the cumulative sums of an fGn sample, where the fGn sample can be generated using algorithms to simulate general stationary Gaussian processes with a given covariance function. Among several existing algorithms, we adopt the simulation algorithm first mentioned by Davies and Harte (1987) and analyzed in Wood and Chan (1994), which is of order $n \log n$ for a sample size of $n$. With the Davies-Harte method, an fGn sample of size $n$ can be constructed as follows. Note that this algorithm requires that $n$ be a power of two:

1. Let $n$ be a power of 2 . For $j=0,1, \ldots, n$, the exact spectral power expected for this autocovariance functions $S_{j}$ is computed, from the discrete Fourier transform of the following sequence of $\gamma_{\alpha}$ : $\gamma_{\alpha}(0), \gamma_{\alpha}(1), \ldots, \gamma_{\alpha}(n-1), \gamma_{\alpha}(n)$

$$
S_{j}=\sum_{m=0}^{n} \gamma_{\alpha}(m) \mathrm{e}^{-\frac{i 2 \pi j m}{2 n}}+\sum_{m=n+1}^{2 n-1} \gamma_{\alpha}(2 n-m) \mathrm{e}^{-\frac{i 2 \pi j m}{2 n}} .
$$

2. It is checked that $S_{j} \geq 0$ for all $j$. This is true for a fractional Gaussian process, negativity indicating that the sequence is corrupt.

3. Let $\left\{Z_{k}, k=0,1, \ldots, 2 n-1\right\}$, be a set of iid Gaussian random variables with zero mean and unit 
variance. Now the randomized spectral amplitudes $V_{k}$ are calculated:

$$
V_{k}= \begin{cases}\sqrt{S_{0}} Z_{0}, & \\ \sqrt{S_{k}}\left(Z_{2 k-1}+i Z_{2 k}\right), & 1 \leq k \leq n-1, \\ \sqrt{S_{n}} Z_{2 n-1}, & n+1 \leq k \leq 2 n-1, \\ V_{2 n-k}^{*}, & \end{cases}
$$

where $*$ denotes the complex conjugate.

4. The simulated realization of the fGn $Y_{j}$ is computed, using the first $n$ elements of the discrete Fourier transform of $V$ :

$$
Y_{j}=\frac{1}{\sqrt{2 n}} \sum_{k=0}^{2 n-1} V_{k} \mathrm{e}^{-\frac{i 2 \pi k j}{2 n}}, \quad j=0,1, \ldots, n-1
$$

Then an fBm sample on an arbitrary equispaced grid is obtained by (2.1). The Davies-Harte algorithm has usually been used for the simulation of fGn with $\alpha \geq 1$ but more recently Craigmile (2003) showed this algorithm is valid for $0<\alpha<1$ too.

\section{Simulation of $\mathcal{H}_{\alpha}$}

Let $\theta_{n, \alpha}=\mathrm{P}\left\{\max _{k \geq 1}\left(B_{\alpha}(k / n)-(k / n)^{\alpha}\right)+\eta \leq 0\right\}$. For simplicity we suppress $\alpha$ of $\theta_{n, \alpha}$ in this section if there is no confusion. According to the representation given in Theorem $1 \mathcal{H}_{\alpha}$ is the limit of the sequence $\mathcal{H}_{n, \alpha}\left(=n \theta_{n}\right)$. For the simulation, $\mathcal{H}_{n, \alpha}$ is truncated as $\mathcal{H}_{n, \alpha}(L)\left(=n \theta_{n}(L)\right)$ for fixed $n$ and $L$, where $\theta_{n}(L)=\mathrm{P}\left\{\max _{1 \leq k \leq n L}\left(B_{\alpha}(k / n)-(k / n)^{\alpha}\right)+\eta \leq 0\right\}$. For each $\alpha$ We estimated $\theta_{n}(L)$ by

$$
\tilde{\theta}_{n}(L)=\frac{1}{2 M} \sum_{m=1}^{M}\left[\left(1-e^{\zeta_{m}(L)}\right) I\left\{\zeta_{m}(L) \leq 0\right\}+\left(1-e^{\tilde{\zeta}_{m}(L)}\right) I\left\{\tilde{\zeta}_{m}(L) \leq 0\right\}\right],
$$

where $\left\{\zeta_{m}(L)\right\}$ are the $M$ independent realizations of $\zeta(L)=\max _{0<l \leq n L}\left\{n^{-\alpha / 2} \sum_{j=1}^{l} Y_{j}-(l / n)^{\alpha}\right\}$ for fractional Gaussian noises $\left\{Y_{j}, j=1,2, \ldots\right\}$ with covariance $\gamma_{\alpha}(j)=|j+1|^{\alpha}+|j-1|^{\alpha}-2|j|^{\alpha}, j \in \mathbb{Z}$, and $\tilde{\zeta}_{m}(L)$ is the $\zeta_{m}(L)$ with $-Y_{j}$ in place of $Y_{j}, j=1,2, \ldots, n L$. The $\tilde{\theta}_{n}(L)$ is the antithetic variates version of

$$
\hat{\theta}_{n}(L)=\frac{1}{M} \sum_{m=1}^{M}\left\{\left(1-e^{\zeta_{m}(L)}\right) I\left(\zeta_{m}(L) \leq 0\right)\right\}
$$

The fact that $\sum_{j=1}^{l} Y_{j} \stackrel{d}{=}-\sum_{j=1}^{l} Y_{j}$ allows us to apply the antithetic variates method so that the standard error and the computing time can be reduced. Thus the approximate value of $\mathcal{H}_{\alpha}, \mathcal{H}_{n, \alpha}(L)$ was estimated by simulating $n \tilde{\theta}_{n}(L)$.

Note that $n \hat{\theta}_{n}(L)$ is an unbiased estimator of $\mathcal{H}_{n, \alpha}(L)$ since we have

$$
\mathrm{E}\left\{\hat{\theta}_{n}(L)\right\}=\mathrm{E}\left\{\left(1-e^{\zeta(L)}\right) I(\zeta(L) \leq 0)\right\}=\int_{0}^{\infty} \mathrm{P}(\zeta(L) \leq-x) e^{-x} d x=\mathrm{P}\{\zeta(L)+\eta \leq 0\}=\theta_{n}(L)
$$


by integration by parts and the unbiasedness is inherited to $n \tilde{\theta}_{n}(L)$. We also have

$$
\begin{aligned}
\operatorname{Var}\left\{\hat{\theta}_{n}(L)\right\} & =\frac{1}{M} \operatorname{Var}\left\{\left(1-e^{\zeta(L)}\right) I(\zeta(L) \leq 0)\right\} \\
& =\frac{1}{M}\left[\mathrm{E}\left\{\left(1-e^{\zeta(L)}\right)^{2} I(\zeta(L) \leq 0)\right\}-\theta_{n}(L)^{2}\right] \\
& =\frac{1}{M}\left[\mathrm{E}\left\{\left(1-e^{\zeta(L)}\right) I(\zeta(L) \leq 0)-e^{\zeta(L)}\left(1-e^{\zeta(L)}\right) I(\zeta(L) \leq 0)\right\}-\theta_{n}(L)^{2}\right] \\
& =\frac{1}{M}\left[\theta_{n}(L)\left(1-\theta_{n}(L)\right)-\mathrm{E}\left\{e^{\zeta(L)}\left(1-e^{\zeta(L)}\right) I(\zeta(L) \leq 0)\right\}\right],
\end{aligned}
$$

which is smaller than $\theta_{n}(L)\left(1-\theta_{n}(L)\right) / M$, the variance of a naive unbiased estimator $\sum_{m=1}^{M} I\left(\zeta_{m}(L)+\right.$ $\left.\eta_{m} \leq 0\right) / M$ of $\theta_{n}(L)$ for unit mean exponential r.v.'s $\eta_{m}$. Thus $\tilde{\theta}_{n}(L)$ also has smaller variance than the naive estimator since it is the antithetic variate version of $\hat{\theta}_{n}(L)$. Moreover, The $\tilde{\theta}_{n}(L)$ has another advantage compared to naive one that the generation of exponential random variables $\eta$ is not necessary.

For the simulation, we chose $n, L, M$, where $L$ defines a cutoff in the maximum, $1 / n$ the grid gap and $M$ the length of the sequence $\zeta_{m}$. We set $n$ as $2^{10}$. Note that the variance of $n \tilde{\theta}_{n}(L)$ is a multiple of $n^{2} / M$. In order to reach a certain accuracy of simulation, larger $M$ should be set for larger $n$ in such a way that $n^{2} / M=o(1)$ but it could not be set sufficiently large because simulation would need excessive computer memory for large $n$. Thus we set $M=50 n^{2}$ for the simulation. The subtracted term $(l / n)^{\alpha}$ in the expression $n^{-\alpha / 2} \sum_{j=1}^{l} Y_{j}-(l / n)^{\alpha}$ increases more sharply at larger $\alpha$ as $l$ increases. Hence we checked where the maximum values are located in the 100 sample paths for each $\alpha$ and chose larger $L$ for smaller $\alpha$. Since it does seem difficult to set $L$ properly for small $\alpha$ due to the erratic sample path and the slow increasing of the $(l / n)^{\alpha}$, we investigated the problem for $\alpha=0.8,0.9, \ldots, 1.9,2$. The simulation experiments were carried out using MATLAB software.

Our estimates $n \tilde{\theta}_{n}(L)$ for $\mathcal{H}_{n, \alpha}(L)$ (denoted $\left.\mathcal{H}_{n, \alpha}(L)-\mathrm{H}\right)$ are presented in Table 1 and plot in Figure 2 as a function of $\alpha$, which are interpolated linearly. Standard errors (SE) are so small as $1.136 \times 10^{-4} \sim$ $9.466 \times 10^{-4}$ for $0.8 \leq \alpha \leq 1.9$. The result indicates that $\mathcal{H}_{\alpha}$ decreases as $\alpha$ increases and the estimated values at $\alpha=1,2$ are around 1 and $1 / \sqrt{\pi}$, respectively. These observations seem to agree with the theoretical facts of the continuity of $\mathcal{H}_{\alpha}$ in $\alpha$ (Dȩbicki, 2006), $\mathcal{H}_{1}=1$ and $\mathcal{H}_{2}=1 / \sqrt{\pi}$. However, the true value 1 is not contained in the approximately $95 \%$ confidence interval $n \tilde{\theta}_{n}(L) \pm 1.96 \times \mathrm{SE}$ constructed at $\alpha=1$. We might get the estimated value of $\mathcal{H}_{1}$ close to one with good accuracy if we could conduct the simulation in a high-performance computing environment.

\section{Concluding Remarks}

Recently Dieker and Yakir (2014) (DY) estimate $\mathcal{H}_{\alpha}$ by simulation based on their new representation

$$
\mathcal{H}_{\alpha}=\mathrm{E}\left[\frac{\sup _{t \in \mathbb{R}} e^{B_{\alpha}(t)-|t|^{\alpha}}}{\int_{-\infty}^{\infty} e^{B_{\alpha}(t)-|t|^{\alpha}} d t}\right]
$$

and its discretized and truncated version

$$
\mathcal{H}_{\alpha}^{n}(L)=\mathrm{E}\left[\frac{n \sup _{-n L \leq k \leq n L} e^{B_{\alpha}\left(\frac{k}{n}\right)-\left|\frac{k}{n}\right|^{\alpha}}}{\sum_{-n L \leq k \leq n L} e^{B_{\alpha}\left(\frac{k}{n}\right)-\left|\frac{k}{n}\right|^{\alpha}} d t}\right] .
$$


Table 1: Estimated values of $\mathcal{H}_{\alpha}$

\begin{tabular}{|c|c|c|c|c|c|}
\hline \multirow{2}{*}{$\alpha$} & \multicolumn{2}{|c|}{$\mathcal{H}_{n, \alpha}(L)-\mathrm{H}$} & \multicolumn{2}{|c|}{$\mathcal{H}_{\alpha}^{n}(L)$-DY } & \multirow{2}{*}{$\frac{1}{\Gamma(1 / \alpha)}$} \\
\hline & Estimate & $\mathrm{SE}$ & Estimate & SE & \\
\hline 0.8 & 1.0638 & 0.0009 & 1.1184 & 0.0136 & 1.1033 \\
\hline 0.9 & 1.0263 & 0.0008 & 1.0539 & 0.0119 & 1.0560 \\
\hline 1 & 0.9834 & 0.0007 & 0.9947 & 0.0106 & 1.0000 \\
\hline 1.1 & 0.9391 & 0.0006 & 0.9424 & 0.0095 & 0.9421 \\
\hline 1.2 & 0.8962 & 0.0005 & 0.8963 & 0.0084 & 0.8859 \\
\hline 1.3 & 0.8536 & 0.0004 & 0.8533 & 0.0074 & 0.8329 \\
\hline 1.4 & 0.8149 & 0.0003 & 0.8121 & 0.0066 & 0.7837 \\
\hline 1.5 & 0.7758 & 0.0003 & 0.7727 & 0.0058 & 0.7385 \\
\hline 1.6 & 0.7395 & 0.0002 & 0.7342 & 0.0051 & 0.6971 \\
\hline 1.7 & 0.7023 & 0.0002 & 0.6970 & 0.0043 & 0.6593 \\
\hline 1.8 & 0.6647 & 0.0001 & 0.6585 & 0.0035 & 0.6247 \\
\hline 1.9 & 0.6241 & 0.0001 & 0.6176 & 0.0025 & 0.5931 \\
\hline 1.998 & 0.5718 & 0.0001 & 0.5663 & 0.0004 & 0.5647 \\
\hline 2 & 0.5642 & 0.0001 & & & 0.5642 \\
\hline
\end{tabular}

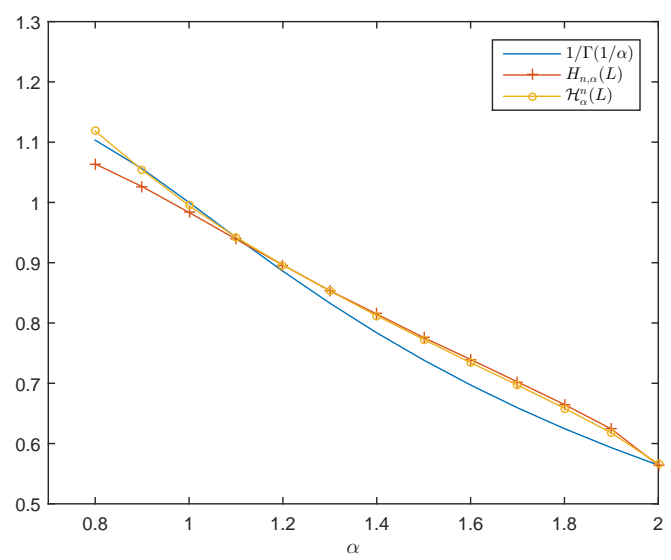

Figure 2: Estimates of $\mathcal{H}_{\alpha}$ as a functions of $\alpha$.

Their simulation was carried out using $L=128$ and $n=2^{18}$ for the cutoff value and the discretization. They improved the computing accuracy by devising a reliable algorithm for estimation. Our approach is based on the relatively simple representation

$$
\mathcal{H}_{\alpha}=\lim _{n \uparrow \infty} n \mathrm{P}\left\{\max _{k \geq 1}\left(B_{\alpha}\left(\frac{k}{n}\right)-\left(\frac{k}{n}\right)^{\alpha}\right)+\eta \leq 0\right\},
$$

and set $n=2^{10}$ and $L=16$ at $\alpha=1.7 \sim 2,32$ at $\alpha=1.2 \sim 1.6,64$ at $\alpha=0.8 \sim 1.1$. Table 1 shows the simulation results based on $\mathcal{H}_{n, \alpha}(L)$ and $\mathcal{H}_{\alpha}^{n}(L)$ (-H and -DY pasted for clarity, respectively) along with the conjectured value $1 / \Gamma(1 / \alpha)$. We obtained the similar values to DY. The differences of the estimated values $H_{n, \alpha}(L)$ and $\mathcal{H}_{\alpha}^{n}(L)$ lie between 0.0001 and 0.0065 for $\alpha>1$. However, our estimates tend to be larger at $\alpha>1.2$ than DY and even exceed the known upper bound $\mathcal{H}_{\alpha}$ for $\alpha>1.8$. This tendency might come from smaller $n$ and $L$ than DY and the difference of representations used for simulation. The standard error of our estimates is smaller than DY, which might not be a fair comparison though since we chose the number of generated sample paths depending on $n$ such as $50 n^{2}$ but DY generated a smaller number of sample paths for estimation. In summary, we see that the representation $\mathcal{H}_{\alpha}^{n}(L)$ 
is more suitable for simulation than $\mathcal{H}_{n, \alpha}(L)$ and DY is more reliable in terms of numerical accuracy at least for $\alpha>1$. However, in mathematical folklore there is a well-known conjecture as follows:

$$
\mathcal{H}_{\alpha}=\frac{1}{\Gamma(1 / \alpha)} .
$$

These hypothetical values are presented in Figure 2 along with the approximated values by simulation of Pickands constants. This conjecture does seem to match the simulated estimates at $\alpha=1$ and 2 and the decreasing property for $\alpha>0.7$. However, the increasing tendency seen in $1 / \Gamma(1 / \alpha)$ for $\alpha$ smaller than about 0.67 is also observed in our additional simulation at $\alpha=0.5,0.6,0.7$ although it is not enough to claim. But we see that the estimated values are above the conjectured curve for $\alpha \geq 1.2$, which was also observed by Dieker and Yakir (2014). Our result serves as supporting evidence that this conjecture is not correct as the simulation of Dieker and Yakir (2014) does. More recently, Harper (2014) theoretically proved that this conjecture is false for small enough $\alpha$.

\section{References}

Adler, R. J. (1990). An Introduction to Continuity, Extreme, and Related Topics for General Gaussian Processes, Institute of Mathematical Statistics, Hayward.

Albin, J. M. P. and Choi, H. (2010). A new proof of an old result by Pickands, Electronic Communications in Probability, 15, 339-345.

Burnecki, K. and Michna, Z. (2002). Simulation of Pickands constants, Probability and Mathematical Statics, 22, 193-199.

Craigmile, P. F. (2003). Simulating a class of stationary Gaussian processes using the Davies-Harte algorithm, with application to long memory processes, Journal of Time Series Analysis, 24, 505511.

Davies, R. B. and Harte, D. S. (1987). Tests for Hurst effect, Biometrika, 14, 95-101.

Dȩbicki, K. (2006). Some properties of generalized Pickands constants, Theory of Probability and Its Applications, 50, 290-298.

Dieker, A. B. and Yakir, B. (2014). On asymptotic constants in the theory of extremes for Gaussian processes, Bernoulli, 20, 1600-1619.

Harper, A. J. (2014). Pickands' constant $H_{\alpha}$ does not equal $1 / \Gamma(1 / \alpha)$, for small $\alpha$, Available from: http://arxiv.org/pdf/1404.5505v1.pdf

Hüsler, J. (1999). Extremes of a Gaussian process and the constant $H_{\alpha}$, Extremes, 2, 59-70.

Leadbetter, M. R., Lindgren, G. and Rootzén, H. (1983). Extremes and Related Properties of Random Sequences and Processes, Springer, New York.

Pickands, J. (1969). Asymptotic properties of the maximum in a stationary Gaussian process, Transactions of the American Mathematical Society, 145, 75-86.

Qualls, C. and Watanabe, H. (1972). Asymptotic properties of Gaussian processes, Annals of Mathematical Statistics, 43, 580-596.

Samoradnitsky, G. and Taqqu, M. S. (1994). Stable Non-Gaussian Random Processes, Chapman \& Hall, New York.

Shao, Q. (1996). Bounds and estimators of a basic constant in extreme value theory of Gaussian processes, Statistica Sinica, 6, 245-257.

Wood, A. T. A. and Chan, G. (1994). Simulation of stationary Gaussian processes in $[0,1]^{d}$, Journal of Computational and Graphical Statistics, 3, 409-432. 